\title{
Improvement of the characterization of the proton source for the European Spallation Source by means of optical emission spectroscopy
}

\author{
M. Mazzaglia, G. Castro, D. Mascali, L. Celona, L. Neri, G. Torrisi, and S. Gammino \\ INFN Laboratori Nazionali del Sud, via S. Sofia 62, 95123 Catania, Italy \\ R. Reitano and E. Naselli \\ INFN Laboratori Nazionali del Sud, via S. Sofia 62, 95123 Catania, Italy \\ and Universitá degli studi di Catania, Dipartimento di Fisica e Astronomia, \\ via S. Sofia 64, 95123 Catania, Italy
}

(Received 15 November 2018; published 6 May 2019)

\begin{abstract}
At the Istituto Nazionale di Fisica Nucleare-Laboratori Nazionali del Sud (INFN-LNS), the commissioning of the high intensity proton source for the European Spallation Source (PS-ESS) has been recently completed. Optical emission spectroscopy measurements carried out with PS-ESS permitted, in a noninvasive way, the evaluation of the electron density, temperature, and relative abundances of the neutral components of a hydrogen plasma. This approach is helpful in finding the optimal source parameters exploiting protons or $H_{2}^{+}$beams production.
\end{abstract}

DOI: 10.1103/PhysRevAccelBeams.22.053401

\section{INTRODUCTION}

A $2.45 \mathrm{GHz}-0.1 \mathrm{~T}$ microwave discharge proton source has been designed and assembled at Istituto Nazionale di Fisica Nucleare-Laboratori Nazionali del Sud (INFN-LNS) as an injector for the proton source for the European Spallation Source (PS-ESS) in order to produce pulsed proton beams from 40 to $90 \mathrm{~mA}$ nominal current, at $75 \mathrm{keV}$ energy and $2.25 \pi \mathrm{mm}$ mrad maximum normalized emittance [1]. The characterization of the proton beam has been performed by means of a Faraday cup, an emittance measurement unit and a Doppler shift measurement unit to determine the proton fraction of the extracted beam. The fraction of protons and $\mathrm{H}_{2}^{+}$of the extracted beam depends on the source parameters such as magnetic field profile, neutral pressure, microwave power, and frequency and also on the dimensions of the plasma chamber [2]. Doppler shift allows the measurement of the in-beam proton fraction only after the ion component has been extracted from the plasma. This implies that current diagnostics do not enable one to obtain direct information about plasma composition and relative abundances of in-plasma species. Still debated, in fact, is the interplay between plasma parameters such as density and electron temperature and the properties of the extracted beam. Measurements of both output beam

Published by the American Physical Society under the terms of the Creative Commons Attribution 4.0 International license. Further distribution of this work must maintain attribution to the author(s) and the published article's title, journal citation, and DOI. properties and plasma parameters are needed. Optical emission spectroscopy (OES) allows the direct "in plasma" measurement of the electron density and temperature, and of the relative abundances of the not-ionized components, such as atomic vs molecular species ratio (in an hydrogen plasma, the ratio between $H$ atoms and $H_{2}$ molecules). Furthermore, OES can be applied online, during routine source operations. OES has therefore been applied to characterize the hydrogen plasma generated by the PS-ESS in optimal experimental configurations, with and without the extraction voltage.

\section{EXPERIMENTAL SETUP}

The PS-ESS is based on the ECR ion sources scheme and designed with a flexible magnetic system consisting of three coaxial copper coils. Thus, the axial magnetic field profile can be tuned in order to optimize the source's performance. The magnetic field profile used for performing the OES measurements hereby illustrated is shown in Fig. 1.

It is well known that quasiflat- $B$ field profiles allow electron cyclotron resonances to occur in two points within the plasma chamber. In the experimentally found optimal magnetic profile, $B$ goes below the corresponding ECR value at $2.45 \mathrm{GHz}$ (i.e., $875 \mathrm{Gs}$ ) for a few millimeters near the injection endplate of the plasma chamber. Figure 2 shows a schematic diagram of the PS-ESS, including the rf power injection system, the three magnetic coils, and the OES diagnostics system. The latter consists of an ImSpector V8E spectrometer, coupled to an acA2040 Complementary Metal Oxide Semiconductor camera.

The spectrometer is sensitive in a spectral range of $380-1000 \mathrm{~nm}$. Its nominal resolution is $2 \mathrm{~nm}$, in agreement 


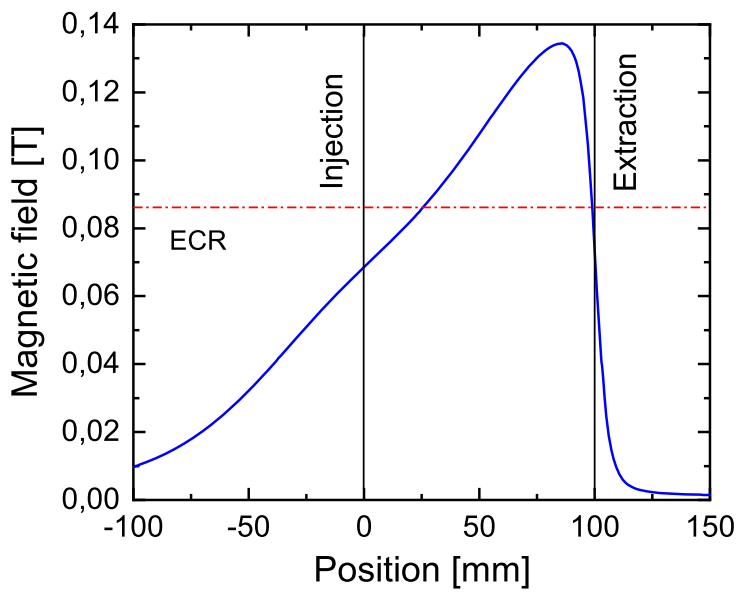

FIG. 1. PS-ESS magnetic field profile that was used during the measurements.

with the measured one. The whole system is connected to the PS-ESS by means of a $1500 \mu \mathrm{m}$ diameter fiberglass that is, in turn, connected to a quartz window allowing the internal inspection of the plasma chamber. The angular aperture of the cone of view of the optical fiber is around $14^{\circ}$ : the light emitted by the plasma is thus integrated over the cone of view volume. The spectrometer was formerly calibrated in relative, using a tungsten filament lamp. This implies that the absolute intensity of an emission line cannot be measured, but only the ration of the lines is a reliable value. PS-ESS has been operated in the most performing (in terms of output currents and proton fraction in the beam) experimental configuration found during the commissioning phase, acquiring data by OES during a rf power scan, while fixing the magnetic field and neutrals pressure. The pressure was $2.7 \times 10^{-5} \mathrm{mbar}$ (this value was measured in the low energy beam transport line, while the pressure value in the plasma chamber can be retrieved by numerical simulations, and it lies in the order of $\sim 3 \times 10^{-3} \mathrm{mbar}$ ); the microwave power was increased from 120 to $1200 \mathrm{~W}$.

PS-ESS is usually operated at $75 \mathrm{kV}$ extraction voltage. However, since sparks may occur from time to time, thus damaging the instrumentation which needs to be placed at

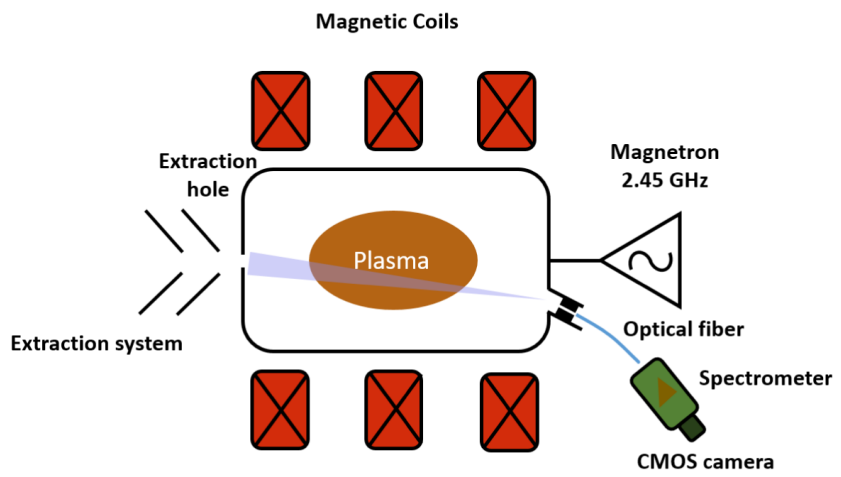

FIG. 2. Schematic of the PS-ESS experimental setup at the INFN-LNS. high voltage, we opted to perform most of the measurements without any extraction voltage, and only a few points (in order to compare them with the voltage-off condition) were taken when extracting the beam. All the data shown below have to be considered in voltage-off mode; at the end, in a table, we show some comparisons with the most significant experimental points, in voltage on and off configurations.

\section{EXPERIMENTAL RESULTS}

Electron density $n_{e}$, electron temperature $T_{e}$, and relative abundances between atomic and molecular hydrogen $n_{H} / n_{H_{2}}$ have been estimated by comparing the line ratio measured during the experimental campaign with the theoretical line ratio estimated by means of a collisional radiative (CR) model. This method is usually known as the "line ratio" method and has been fully described in Refs. $[3,4]$.

Figure 3 shows a typical hydrogen spectrum acquired during measurements (after background subtraction). Lines of interest for the line ratio method application are the Balmer series of atomic hydrogen $\left(H_{\alpha}\right.$ to $\left.H_{\gamma}\right)$ and the Fulcher- $\alpha$ transition of the $H_{2}$ molecule $\left(d^{3} \Pi_{u} \rightarrow a^{3} \Sigma_{u}^{+}\right)$.

$H_{\beta} / H_{\gamma}$ and $H_{\alpha} / H_{\beta}$ theoretical line ratios in the density and temperature range of our interest are calculated from Yacora [5]. Experimental line ratios are then compared with theoretical line ratios by solving the following nonlinear equations system:

$$
\begin{aligned}
& {\left[\frac{H_{\beta}}{H_{\gamma}}\right]_{\exp }=\left[\frac{H_{\beta}}{H_{\gamma}}\right]_{\mathrm{th}}\left(n_{e}, T_{e}\right)} \\
& {\left[\frac{H_{\alpha}}{H_{\beta}}\right]_{\exp }=\left[\frac{H_{\alpha}}{H_{\beta}}\right]_{\mathrm{th}}\left(n_{e}, T_{e}\right) .}
\end{aligned}
$$

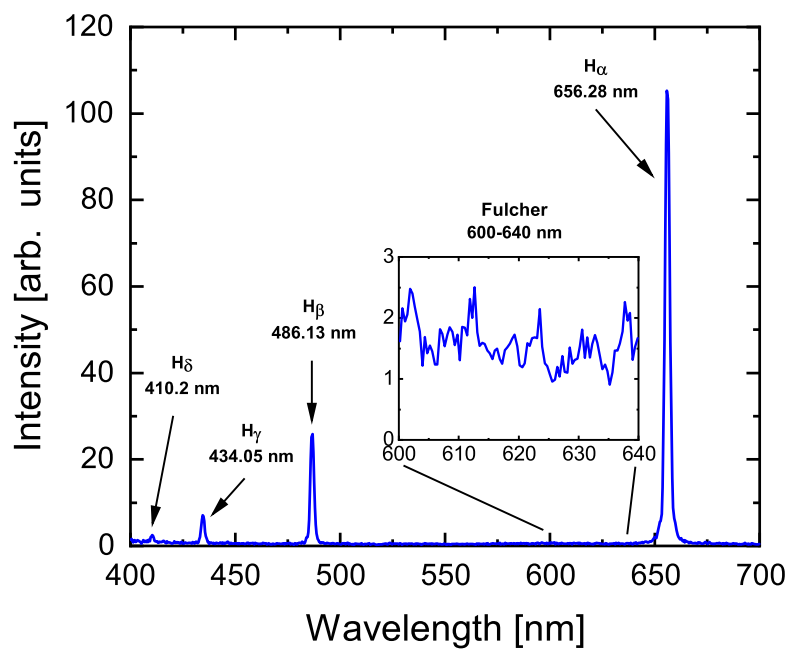

FIG. 3. Hydrogen spectrum acquired at $400 \mathrm{~W}$ microwave power, $2.45 \mathrm{GHz}$ microwave frequency,and at pressure of $2.7 \times 10^{-5}$ mbar. Balmer line series and Fulcher band are highlighted. 


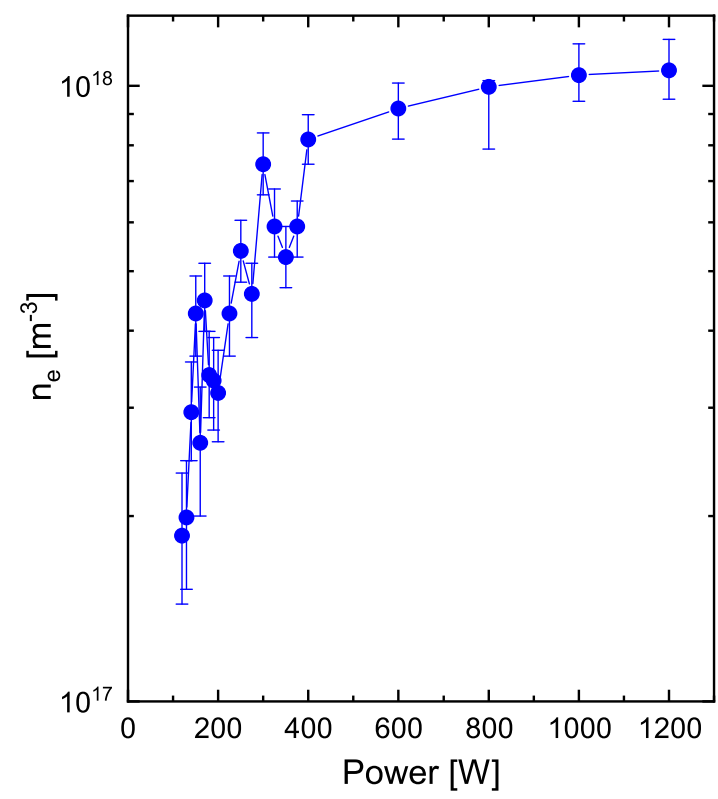

FIG. 4. Electron density evaluated via OES when increasing the rf power.

The solution of Eq. (1) allows the simultaneous determination of the electron density and temperature. Furthermore, $n_{H} / n_{H_{2}}$ ratio has been obtained by the ratio between $H_{\gamma}$ and the integral of the Fulcher band (multiplied by two [4]). Figure 4 shows the estimated electron density $n_{e}$, in units of $\mathrm{m}^{-3}$, as a function of microwave power, in logarithmic scale. $n_{e}$ increases with rf power from $\simeq 1 \times$ $10^{17} \mathrm{~m}^{-3}$ at $120 \mathrm{~W}$ to $\simeq 1 \times 10^{18} \mathrm{~m}^{-3}$ above $800 \mathrm{~W}$.

For any rf power, the measured density is larger than the cutoff density at $2.45 \mathrm{GHz}$, corresponding to $6.85 \times 10^{16}$ $\mathrm{m}^{-3}$. The experimental evidence of overdense plasma generation deserves further consideration. The occurrence of overdense plasmas in microwave discharges operated around $2.45 \mathrm{GHz}$ has been verified by many experiments and other authors [6-8]. Although no overall explanation is currently available, the most plausible ones are (i) the evanescent wave, propagating for several $\mathrm{cm}$ in the overdense plasma, and (ii) alternative mechanisms of waveto-plasma coupling. The electron Bernstein wave (EBW) heating at the upper hybrid resonance is among them, being EBW able to propagate in plasmas of whatever density [9]. Experiments performed on a device very similar to the proton source described in the present paper demonstrate this mechanism is effective in supporting very overdense plasmas [10].

Electron temperature $T_{e}$, in $\mathrm{eV}$ units, is shown in Fig. 5: it ranges between 5 and $15 \mathrm{eV}$, showing a slight decrease at microwave power larger than $300 \mathrm{~W}$. A similar trend, obtained by means of Langmuir probe measurements, has been reported in [11].

An option for explaining this decreasing (and, somehow, unexpected) trend of $T_{e}$ is to argue a thermal coupling

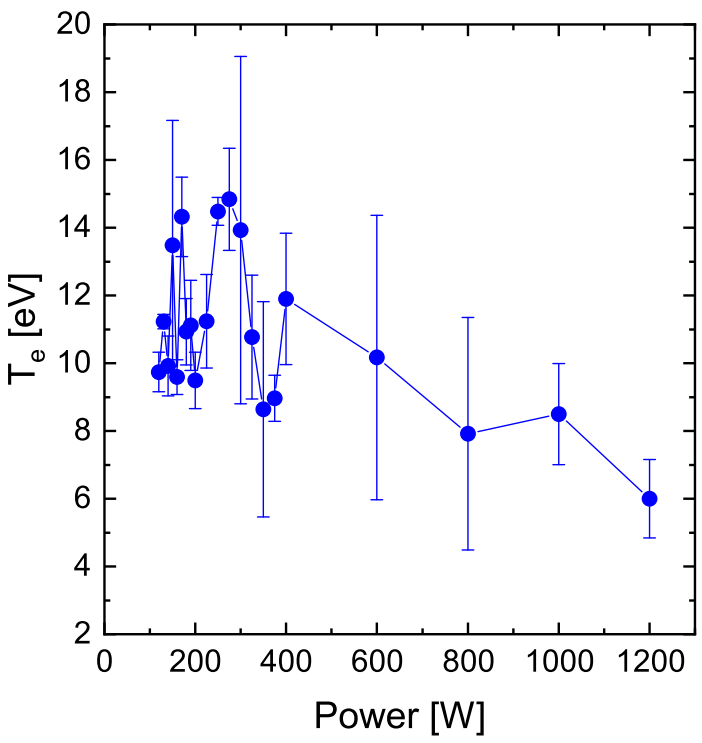

FIG. 5. Electron temperature evaluated via OES when increasing the $\mathrm{rf}$ power.

between cold ions and warm electrons as the microwave power increases, mediated by the exponential increase of the electron density up to 350-400 W. In principle, microwave heating acts only on the electron population, while ions should remain cold. Electron-ion collisions, however, can transfer energy from the electron to the ion population, as their frequency increases linearly with the electron density. Thus, the energy transfer can occur (i.e., electrons become colder, causing ions to warm up) if the characteristic time for energy transfer between ions and electrons $\tau_{m}^{e i}$ is smaller than the mean ion lifetime in plasma $\tau_{i} . \tau_{m}^{e i}$ has been calculated by considering the Spitzer collisions as the main mechanism of energy transfer from electrons to ions.

Time needed for electron-ion Spitzer collisions $\tau_{s p}$ depends on both $n_{e}$ and $T_{e}$ as follows [7]:

$$
\tau_{s p}^{-1} \sim 2 \times 10^{-6} \mathrm{Zn}\left(\mathrm{cm}^{-3}\right) \ln \left[\Lambda_{D} / b\right] / T_{e}^{3 / 2}(\mathrm{eV})
$$

where $\ln \left[\Lambda_{D} / b\right]$ is the Coulomb logarithm ranging between 10 and 20 [7] and $Z$ is the ion charge. $\tau_{m}^{e i}$ is obtained by $\tau_{s p}$ by the following equation:

$$
\tau_{m}^{e i} \sim \frac{m_{i}}{m_{e}} \tau_{90^{\circ}}^{e i}
$$

$\tau_{m}^{e i}$ has been estimated for plasma parameters obtained in the experimental campaign (Fig. 6).

The plot shows that $\tau_{m}^{e i}$ decreases as microwave power increases. In particular, it decreases from 4-8 ms to less than $1 \mathrm{~ms}$ at higher power. Estimated typical ion lifetime in a microwave discharge proton source is $\sim 0.1 / 1 \mathrm{~ms}$; it follows that at high power regimes, plasma density and temperature make $\tau_{m}^{e i}$ comparable with $\tau_{i}$. Consequently, 


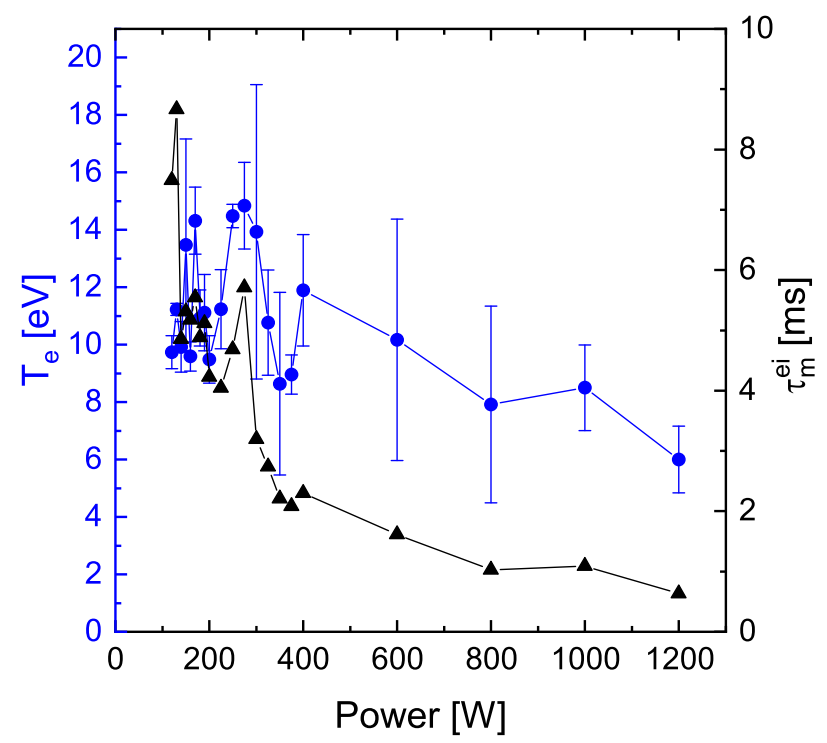

FIG. 6. Comparison between the trend of $T_{e}$ vs the $\mathrm{rf}$ power with the corresponding energy transfer average time, as determined by Eq. (3).

data are compatible with an energy exchange between electrons and ions at increasing microwave power, leading to the cooling of the electron population. As a consequence, an increase of the ion temperature should be measured in the same experimental conditions, but ion temperature estimation is, for the moment, beyond the capabilities of our experimental setup.

Finally, Fig. 7 shows the density ratio of atomic to molecular hydrogen inside plasma vs power. $n_{H} / n_{H_{2}}$ increases with microwave power from $\sim 0.5$ to $\sim 2$.

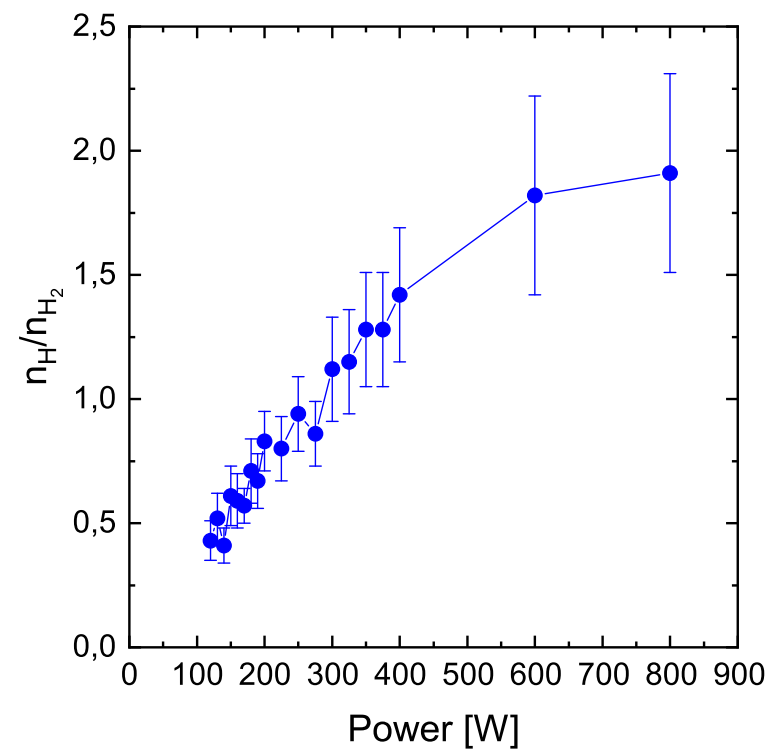

FIG. 7. Density ratio of atomic to molecular hydrogen obtained from the OES evaluations for varying power.

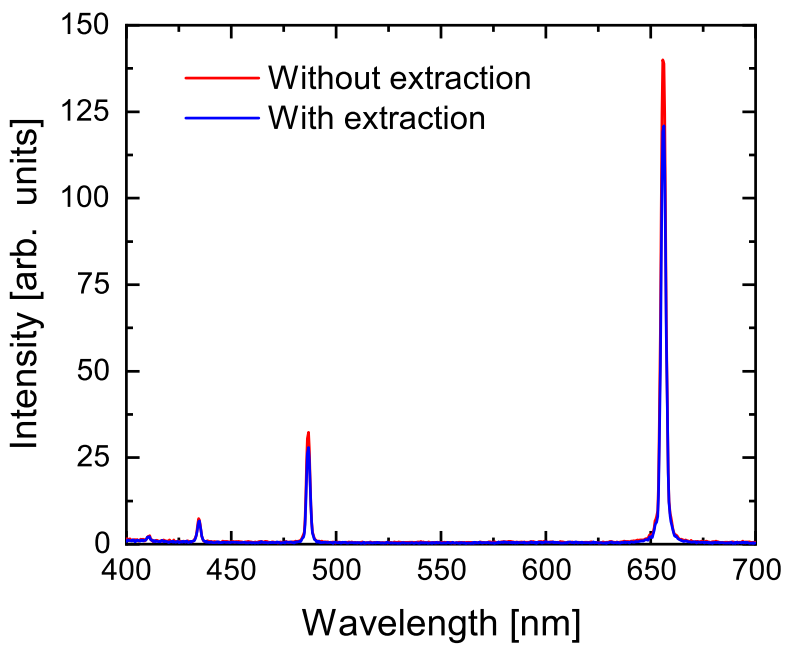

FIG. 8. Hydrogen spectrum obtained at $400 \mathrm{~W}$ without and with beam extraction (i.e., with the $75 \mathrm{kV}$ voltage set on and off).

In the second part of the experiment, a $75 \mathrm{kV}$ extraction voltage has been applied to PS-ESS. Measurements have been carried out in the same experimental conditions as before, but only for three values of microwave power: 200 400 and 600 W. Figure 8 shows a hydrogen spectrum obtained at $400 \mathrm{~W}$ without and with $75 \mathrm{kV}$ extraction voltage.

It is clear from the acquired spectra that the application of the extraction voltage leads to a decrease of the light emission from the plasma. Although the extraction voltage cannot penetrate the plasma because of the Debye shielding, it is evident that plasma is anyway perturbed by the external voltage application.

Table I shows the comparison between plasma parameters obtained with and without the beam extraction. Plasma parameters are modified by the extraction voltage application. In particular, at $200 \mathrm{~W}$ electron density and temperature are larger in the voltage-off mode, while the species density ratio is almost the same. At 400 and $600 \mathrm{~W}$ all the measured parameters are lower when no extraction is applied. These results will require more time for a correct

TABLE I. Plasma parameters with and without beam extraction.

\begin{tabular}{|c|c|c|c|c|}
\hline & $\begin{array}{c}\text { RF power } \\
{[\mathrm{W}]}\end{array}$ & $n_{H} / n_{H_{2}}$ & $n_{e}\left[m^{-3}\right] \times 10^{17}$ & $T_{e}[\mathrm{eV}]$ \\
\hline \multirow{3}{*}{$\begin{array}{l}\text { Without } \\
\text { extraction }\end{array}$} & 200 & $0.83 \pm 0.12$ & $3.17_{-0.53}^{+0.55}$ & $9.49 \pm 0.83$ \\
\hline & 400 & $1.42 \pm 0.27$ & $8.18_{-0.72}^{+0.79}$ & $11.90 \pm 1.94$ \\
\hline & 600 & $1.82 \pm 0.40$ & $9.19_{-1}^{+0.91}$ & $10.17 \pm 4.20$ \\
\hline \multirow{3}{*}{$\begin{array}{l}\text { With } \\
\text { extraction }\end{array}$} & 200 & $0.75 \pm 0.13$ & $4.48_{-0.58}^{+0.79}$ & $14.32 \pm 1.17$ \\
\hline & 400 & $1.09 \pm 0.19$ & $6.19_{-0.67}^{+0.77}$ & $9.74 \pm 1.67$ \\
\hline & 600 & $1.27 \pm 0.24$ & $6.96_{-0.77}^{+0.68}$ & $7.30 \pm 0.59$ \\
\hline
\end{tabular}


interpretation. For the moment, we can state that experimental results clearly demonstrate that the cone-of-view averaged values of plasma density and electron temperature are changing in voltage on/off modes, but no data are available about the density and temperature profiles. In principle, the different values in on/off modes may be due to changes in the plasma profile, since the application of the voltage modifies the plasma diffusion processes towards the plasma chamber walls. One possible mechanism could be the following: when extraction voltage is applied, ions are extracted from the plasma, while electrons are reflected back. In order to compensate such fluxes, to keep quasineutrality, plasma then modifies its losses by changing radial and axial density gradients, hence the density profiles.

\section{CONCLUSIONS AND PERSPECTIVES}

We have reported an optical emission spectroscopy characterization of a hydrogen plasma in a high intensity proton source. The line ratio method has allowed us to simultaneously determine electron density and temperature, along with relative atomic and molecular hydrogen abundances. The measurements aimed at measuring the PS-ESS source parameters in the best (experimentally found) operating conditions, i.e., when more than $100 \mathrm{~mA}$ total current with $90 \%$ proton fraction is extracted. The investigation of the plasma density, temperature, and species ratio as a function of the microwave power has allowed a direct comparison with experimentally determined trends of the extracted current and beam species. In particular, the measurements have permitted us to measure an electron density up to almost $1 \times 10^{18} \mathrm{~m}^{-3}$, for $P_{r f}>800 \mathrm{~W}$, i.e., a value around ten times the corresponding cutoff density at $2.45 \mathrm{GHz}$. The decreasing trend of temperature at the highest power levels was tentatively explained by taking into account a thermal coupling between the ion and electron population at larger and larger densities.

Measurements with and without extraction voltage have shown that the plasma parameters, averaged over the cone of view of the optical fiber, depend on the application of the extraction voltage. The modification of measured plasma parameters could be due to changes in density and temperature profiles induced by the loss fluxes modifications caused by the ion extraction. This means that further measurements should focus on high voltage conditions, in order to more deeply understand plasma properties in voltage on and off modes.

The next step is to find a close relationship between plasma parameters and beam parameters, as proton fraction and beam intensity. This could permit one to monitor, online and in a noninvasive way, the beam parameters by only using OES diagnostics. Present diagnostics do not permit one to make any consideration about beam emittance, since it depends on the ion temperature which cannot be currently measured. However, commissioning of two different OES detectors in next months will permit a more accurate investigation of plasma parameters. The first is a $40 \mathrm{pm}$ monochromator detector. Its resolution is enough to characterize rotational temperature of a molecule [12] and to obtain higher resolution emission lines, which is important for improving hydrogen plasma studies and extending them to other plasmas, where high resolution is required (i.e., Argon). The second is Spettrometro Alta Risoluzione Galileo, a powerful spectropolarimeter formerly installed at TNG, Telescopio Nazionale Galileo, Canary Islands, and now installed at INFN-LNS. Spettrometro Alta Risoluzione Galileo allows to reach very high resolution: $\mathrm{R}=160.000$ in the range 370-900 $\mathrm{nm}$, suitable for ion temperature measurements and/or online discrimination of the ionization states of the ions inside the plasma.

\section{ACKNOWLEDGMENTS}

The support of the 5th National Committee of INFN through the PANDORA experiment and the ESS-MIUR experiment is gratefully acknowledged. The contribution of A. Miraglia, F. Chines, and of the INFN-LNS mechanics staff is warmly acknowledged. The contributions and suggestions of U. Fantz and S. Briefi have been essential for the preparation of the present work.

[1] L. Neri et al., Beam commission of the high intensity proton source developed at INFN-LNS for the European Spallation Source, J. Phys. Conf. Ser. 874, 012037 (2017).

[2] G. Castro, D. Mascali, R. Agnello, L. Celona, O. Leonardi, L. Neri, D. Nicolosi, G. Torrisi, and S. Gammino, Experimental investigation of non-linear wave to plasma interaction in a quasi-flat magnetostatic field, Rev. Sci. Instrum. 87, 02A507 (2016).

[3] U. Fantz, Basics of plasma spectroscopy, Plasma Sources Sci. Technol. 15, S137 (2006).

[4] U. Fantz, H. Falter, P. Franzen, D. Wünderlich, M. Berger, A. Lorenz, W. Kraus, P. McNeely, R. Riedl, and E. Speth, Spectroscopy - a powerful diagnostic tool in source development, Nucl. Fusion 46, S297 (2006).

[5] D. Wunderlich and U. Fantz, Evaluation of state-resolved reaction probabilities and their application in population models for $\mathrm{He}, \mathrm{H}$, and $\mathrm{H}_{2}$, Atom 4, 26 (2016).

[6] G. Lisitano, M. Fontanesi, and E. Sindoni, Nonresonant absorption of electromagnetic waves in a high-density plasma, Appl. Phys. Lett. 16, 122 (1970).

[7] R. Geller, Electron Cyclotron Resonance Ion Sources and ECR Plasmas (Institute of Physics Publishing, Bristol, 1996).

[8] G. Castro et al., Comparison between off-resonance and electron Bernstein waves heating regime in a microwave discharge ion source, Rev. Sci. Instrum. 83, 02B501 (2012).

[9] I. B. Bernstein, Waves in a plasma in a magnetic field, Phys. Rev. 109, 10 (1958). 
[10] G. Castro et al., Overdense plasma generation in a compact ion source, Plasma Sources Sci. Technol. 26, 055019 (2017).

[11] P. Roychowdhury and D. P. Chakravarthy, High intensity electron cyclotron resonance proton source for low energy high intensity proton accelerator, Rev. Sci. Instrum. 80, 123305 (2009).
[12] S. Briefi, D. Rauner, and U. Fantz, Determination of the rotational population of $\mathrm{H}_{2}$ and $\mathrm{D}_{2}$ including high- $N$ states in low temperature plasmas via the Fulcher- $\alpha$ transition, J. Quant. Spectrosc. Radiat. Transfer 187, 135 (2017). 